\title{
Mechanics of Supercooled Liquids
}

\section{Citation}

Li, Jianguo, Qihan Liu, Laurence Brassart, and Zhigang Suo. 2014. "Mechanics of Supercooled Liquids." J. Appl. Mech. 81 (11) (September 24): 111007.

\section{Published Version}

doi:10.1115/1.4028587

\section{Permanent link}

http://nrs.harvard.edu/urn-3:HUL.InstRepos:13363864

\section{Terms of Use}

This article was downloaded from Harvard University's DASH repository, and is made available under the terms and conditions applicable to Open Access Policy Articles, as set forth at http:// nrs.harvard.edu/urn-3:HUL.InstRepos:dash.current.terms-of-use\#OAP

\section{Share Your Story}

The Harvard community has made this article openly available.

Please share how this access benefits you. Submit a story.

\section{Accessibility}




\title{
Mechanics of Supercooled Liquids
}

\section{Jianguo Li ${ }^{1}$}

School of Engineering and Applied Sciences, Kavli Institute for Bionano Science and Technology, Harvard University, Cambridge, Massachusetts 02138, USA

International Center for Applied Mechanics, State Key Lab for Strength and Vibration of Mechanical Structures, School of Aerospace Engineering, Xi'an Jiaotong University, Xi'an 710049, China

\section{Qihan Liu'}

School of Engineering and Applied Sciences, Kavli Institute for Bionano Science and Technology, Harvard University, Cambridge, Massachusetts 02138, USA

\section{Laurence Brassart}

Institute of Mechanics, Materials and Civil Engineering, Université catholique de Louvain, 1348 Louvain-la-Neuve, Belgium

\section{Zhigang Suo ${ }^{2}$}

School of Engineering and Applied Sciences, Kavli Institute for Bionano Science and Technology, Harvard University, Cambridge, Massachusetts 02138, USA e-mail: suo@seas.harvard.edu ASME Fellow

\begin{abstract}
Pure substances can often be cooled below their melting points and still remain in the liquid state. For some supercooled liquids, a further cooling slows down viscous flow greatly, but does not slow down self-diffusion as much. We formulate a continuum theory that regards viscous flow and self-diffusion as concurrent, but distinct, processes. We generalize Newton's law of viscosity to relate stress, rate of deformation, and chemical potential. The self-diffusion flux is taken to be proportional to the gradient of chemical potential. The relative rate of viscous flow and self-diffusion defines a length, which, for some supercooled liquids, is much larger than the molecular dimension. A thermodynamic consideration leads to boundary conditions for a surface of liquid under the influence of applied traction and surface energy. We apply the theory to a cavity in a supercooled liquid and identify a transition. A large cavity shrinks by viscous flow, and a small cavity shrinks by self-diffusion.
\end{abstract}

\footnotetext{
${ }^{1}$ These authors contributed equally to this work.

${ }^{2}$ Corresponding author.
} 


\section{Introduction}

In a pure liquid, molecules of the same species touch one another but frequently change neighbors. The rate of change has long been studied in experiments of two types. External forces cause the liquid to deform by viscous flow, characterized by the viscosity [1]. Thermal agitation causes individual molecules to migrate by self-diffusion, characterized by the selfdiffusivity [2-4]. In a given liquid, the two kinetic processes often obey the Stokes-Einstein relation:

$$
\frac{\eta D}{k T}=\frac{1}{C b}
$$

where $\eta$ is the viscosity, $D$ the self-diffusivity, $T$ the temperature, $k$ the Boltzmann constant, $b$ a length characteristic of the size of the molecules, and $C$ a dimensionless number depending on the shape of the molecules. As the temperature drops, the liquid increases its viscosity and decreases its self-diffusivity, but the Stokes-Einstein relation predicts that the ratio $\eta D / k T$ is a constant independent of temperature. In his original paper [5], Einstein did not analyze selfdiffusion; rather, he analyzed a macroscopic particle diffusing in a liquid using the continuum theory of Stokes flow. Nonetheless the Stokes-Einstein relation holds for particles down to the molecular scale [6]. In particular, the relation holds for self-diffusion for many liquids over wide ranges of temperature [7].

The Stokes-Einstein relation even holds for some supercooled liquids, substances that remain in the liquid state and do not crystalize when the temperature drops below their melting points [8]. For instance, water has a melting point of $\mathrm{O}^{\circ} \mathrm{C}$, but can remain in the liquid state down to $-37 \cdot 5^{\circ} \mathrm{C}$ [9]. As the temperature drops, a supercooled liquid like silica increases its viscosity and reduces its self-diffusivity, both by orders of magnitude, but keeps the ratio $\eta D / k T$ independent of temperature (Fig. 1).

In recent decades, however, evidence has accumulated that some supercooled liquids violate the Stokes-Einstein relation [10-16]. When the temperature drops, such a supercooled 
liquid increases its viscosity steeply (Fig. 1a), but does not reduce its self-diffusivity as steeply (Fig. 1b), such that the ratio $\eta D / k T$ increases often by orders of magnitude (Fig. 1c).

For a liquid that obeys the Stokes-Einstein relation, viscous flow and self-diffusion proceed through a single rate-limiting process: molecules change neighbors. By contrast, for a liquid that violates the Stokes-Einstein relation, viscous flow and self-diffusion proceed by distinct rate-limiting processes. In many cases a supercooled liquid forms a dynamic structure that consists of regions larger than individual molecules [17-30]. Molecular rearrangement is much slower in some regions than others (Fig. 2). Viscous flow proceeds by disrupting the dynamic structure, but self-diffusion proceeds by the migration of individual molecules through the fast regions. Consequently, as the temperature drops, the dynamic structure increasingly jams viscous flow, but does not retard self-diffusion as much.

As molecular dynamic simulations and microscopic experiments continue to shed light on the physics and chemistry of supercooled liquids, it is timely to formulate a continuum theory of supercooled liquids to describe macroscopic phenomena, and to motivate new experiments. Here we formulate a continuum theory by regarding viscous flow and self-diffusion as distinct, concurrent processes. We generalize Newton's law of viscosity to relate stress, rate of deformation, and chemical potential (Section 2). We assume that the self-diffusion flux is proportional to the gradient of chemical potential (Section 3). The relative rate of viscose flow and self-diffusion results in a characteristic length (Section 4). We apply the theory to a cavity in a supercooled liquid, shrinking under the influence of the surface energy (Section 5). The characteristic length demarcates two types of behavior: a large cavity shrinks by the viscous flow, and a small cavity shrinks by the self-diffusion. We place the theory in the context of several other theories (Section 6).

\section{Homogeneous State}


This section generalizes Newton's law of viscosity to describe a supercooled liquid of a single species of molecules, subject to external forces, and connected to a reservoir of the same species of molecules (Fig. 3). The piece of liquid contains a large number of molecules, but is still small enough to serve as a representative elementary volume. In this continuum picture, the piece evolves through a sequence of homogeneous states, represented by a parallelepiped that changes its shape and volume. Let $d_{i j}$ be the rate of deformation-for example, $d_{11}$ is the rate of extension of the piece of liquid in direction 1 , and $d_{12}$ is the rate of shear of the piece of liquid between directions 1 and 2. Let $R$ be the rate of injection-that is, the number of molecules transferred from the reservoir to the piece per unit volume per unit time. The external forces apply to the piece a state of stress $\sigma_{i j}$, and the molecules in the reservoir have the chemical potential $\mu$.

Molecules in a liquid are often nearly incompressible. As an idealization, we assume that the volume per molecule in the liquid, $\Omega$, remains constant, independent of the stress and the chemical potential of the molecules in the reservoir. At a given time, the volume of the piece is $V$, the trace of the rate of deformation is $d_{k k}$, and the piece changes its volume at the rate $V d_{k k}$. The reservoir transfers volume of molecules to the piece at rate $\Omega R V$. The idealization of molecular incompressibility, along with the conservation of the number of molecules, requires that

$$
d_{k k}=\Omega R .
$$

That is, the increase of the volume of the piece equals the volume transferred from the reservoir.

The piece of liquid, the external forces, and the reservoir together constitute a composite thermodynamic system. The composite system exchanges energy, but not molecules, with a heat bath of a fixed temperature. We restrict our analysis to isothermal processes, and do not list temperature as a variable. Consistent with the idealization of molecular incompressibility, we 
further assume that the Helmholtz free energy per molecule in the piece of liquid remains constant, independent of the applied stress and the chemical potential of the molecules in the reservoir. We set the Helmholtz free energy per molecule in the piece of liquid to be zero. The potential energy of the external forces changes at the rate $-V \sigma_{i j} d_{i j}$, and the potential energy of the reservoir changes at the rate $-V \mu R$. The Helmholtz free energy of the composite system is the sum over the parts (i.e., the piece of liquid, the external forces, and the reservoir of molecules): $0-V \sigma_{i j} d_{i j}-V \mu R$. Thermodynamics requires that the Helmholtz free energy of the composite system should never increase, giving

$$
-V \sigma_{i j} d_{i j}-V \mu R \leq 0
$$

This thermodynamic condition, subject to idealization of molecular incompressibility, $d_{k k}=\Omega R$, becomes that

$$
\left(\sigma_{i j}+\frac{\mu}{\Omega} \delta_{i j}\right) d_{i j} \geq 0 .
$$

Condition (4) holds for arbitrary rate of deformation $d_{i j}$, a total of six independent variables.

When the equality in (4) holds for arbitrary rate of deformation $d_{i j}$, the composite system is in thermodynamic equilibrium. This condition of equilibrium leads to

$$
\sigma_{i j}+\frac{\mu}{\Omega} \delta_{i j}=0 .
$$

This condition corresponds to a total of six independent equilibrium equations, $\sigma_{12}=\sigma_{23}=\sigma_{31}=0$ and $\sigma_{11}=\sigma_{22}=\sigma_{33}=-\mu / \Omega$. For the liquid, the external forces, and the reservoir to equilibrate, the liquid must be in a hydrostatic state, and the hydrostatic stress in the liquid must compensate the chemical potential of molecules in the reservoir.

When the inequality in (4) holds for arbitrary rate of deformation $d_{i j}$, the composite system is not in thermodynamic equilibrium. We satisfy this inequality by prescribing a kinetic 
model that linearly relates two symmetric tensors, $\sigma_{i j}+(\mu / \Omega) \delta_{i j}$ and $d_{i j}$. For an isotropic liquid, the linear relation between two symmetric tensors takes the general form [31]

$$
\sigma_{i j}+\frac{\mu}{\Omega} \delta_{i j}=2 \eta\left(d_{i j}-\frac{1}{3} d_{k k} \delta_{i j}\right)+\beta d_{k k} \delta_{i j} .
$$

where $\eta$ and $\beta$ are constants. The thermodynamic inequality (4) holds when both $\eta$ and $\beta$ are nonnegative. Given the material constants $\Omega, \eta$ and $\beta$, this kinetic model and the constraint (2) together provide a total of seven independent equations among the fourteen variables $d_{i j}, R, \sigma_{i j}$, and $\mu$.

The kinetic model (6) can also be motivated in a different way. The trace of the rate of deformation, $d_{k k}$, describes the rate at which the piece of liquid changes its volume. The deviatoric part of the rate of deformation, $e_{i j}=d_{i j}-d_{k k} \delta_{i j} / 3$, describes the rate at which the piece of liquid changes its shape. The mean stress is $\sigma_{m}=\sigma_{k k} / 3$, and the deviatoric stress is $s_{i j}=\sigma_{i j}-\sigma_{m} \delta_{i j}$. Note the identity $\sigma_{i j} d_{i j}=s_{i j} e_{i j}+\sigma_{m} d_{k k}$, and write (4) as $s_{i j} e_{i j}+\left(\sigma_{m}+\mu / \Omega\right) d_{k k} \geq 0$. This inequality suggests a kinetic model: $s_{i j}=2 \eta e_{i j}$ and $\sigma_{m}+\mu / \Omega=\beta d_{k k}$. The thermodynamic inequality holds when both $\eta$ and $\beta$ are nonnegative. This kinetic model is equivalent to (6).

The constant $\eta$ represents the shear viscosity that resists the change in shape, and the constant $\beta$ represents the bulk viscosity that resists the change in volume. In the limit $\beta / \eta \rightarrow 0$, the change in volume is much faster than change in shape, so that we may assume that $\sigma_{m}+\mu / \Omega \rightarrow 0$, and the composite system is in partial thermodynamic equilibrium with respect to the exchange of molecules between the piece and the reservoir. In the limit $\eta / \beta \rightarrow 0$, the change in shape is much faster than change in volume, so that we may assume that $d_{k k}=0$, and the piece changes shape without changing volume. 
The kinetic model (6) generalizes Newton's law of linear, isotropic, viscous flow. Our model differs from the model of compressible viscosity. We have assumed that molecules in the liquid are incompressible, so that an increase in volume of the liquid is entirely due the injection of molecules from the reservoir to the piece. By contrast, for compressible viscosity, the number of molecules in an element of liquid is fixed, and an increase in the volume of the element is entirely due to the reduction in density.

\section{Inhomogeneous State}

We now consider a body of liquid evolving through a sequence of inhomogeneous states. A continuum theory regards the body as a collection of small pieces. As the body evolves, each piece evolves through a sequence of homogeneous states, as described in the previous section. Different pieces communicate through the balance of forces, compatibility of geometry, and transfer of molecules. In classical hydrodynamics, each small piece (commonly called a material particle, a material point, or a material element) is identified by a set of molecules, which do not change identity as the body of liquid evolves. This practice, however, is inapplicable here. As a small piece of liquid transfers molecules to surrounding small pieces by the self-diffusion of molecules, the small piece does not consist of the same molecules and may even disappear after some time.

Here we break away from this practice in classical hydrodynamics, and describe the kinematics of the body not by tracking pieces of the liquid, but by tracking markers dispersed in the liquid. Indeed, markers are commonly used in experiments. Examples include precipitates that visualize the creep of a solid solution, and particles that visualize the flow of a fluid. The markers should be small compared to the length characteristic of the inhomogeneous field, so that the markers are carried by the flow without affecting it. The markers should be large compared to the size of the individual molecules, so that the markers themselves diffuse negligibly. As common with many basic concepts, the relation between markers in experiments 
and markers in the continuum theory deserves careful examination. This paper proceeds by describing the idealized behavior of the markers within the continuum theory.

We relate the velocity field of markers and the rate of deformation. When two nearby markers drift apart, the piece of the liquid between the markers elongates, and the relative velocity between the two markers divided by the distance between the two markers defines the rate of extension. At a given time, consider three nearby markers $\mathrm{P}, \mathrm{A}$ and $\mathrm{B}$, with the line PA normal to the line PB. When the line PA rotates relative to the line PB, the piece of liquid shears, and the rate of the change in the angle between the two lines defines the rate of shear. Denote the velocity of the markers by a field $\mathbf{v}$. In general, the rate of deformation relates to the gradient of the marker velocity as $d_{i j}=\left(v_{i, j}+v_{j, i}\right) / 2$. Here $d_{11}, d_{22}$ and $d_{33}$ are the rates of extension in the three directions, whereas $d_{23}, d_{31}$ and $d_{12}$ are the rates of shear. As usual, a factor of 2 is introduced in the definitions of $d_{23}, d_{31}$ and $d_{12}$, such that the three rates of shear, along with the three rates of extension, constitute the components of a symmetric, second-rank tensor. This relation between the rate of deformation and the velocity of markers expresses the compatibility of geometry.

Following a practice in materials science [32-34], we identify fluxes of three types (Fig. 4). Markers in a liquid are analogous to leaves on a river. The flow of water carries the leaves, but is unaffected by their presence. The motion of the markers defines convection. The molecular flux in excess of convection defines diffusion. The velocity of markers defines the convective flux, $\mathbf{v} / \Omega$. Consider a plane fixed in space, with $\mathbf{n}$ being the unit vector normal to the plane. Let $\mathbf{N}$ be the net flux of molecules-that is, $N_{i} n_{i}$ is the number of molecules crossing the plane per unit area per unit time. The velocity of markers, $\mathbf{v}$, and the net flux of molecules, $\mathbf{N}$, can be independently measured in macroscopic experiments. Classical hydrodynamics assumes that the net flux equals the convective flux, $\mathbf{N}=\mathbf{v} / \Omega$. The two quantities, however, are unequal 
when pieces of the liquid exchange molecules. The difference between the net flux and the convective flux defines the self-diffusion flux of molecules:

$$
\mathbf{J}=\mathbf{N}-\mathbf{v} / \Omega .
$$

That is, the net flux of molecules is the sum of the self-diffusion flux and the convective flux.

Due to molecular incompressibility, the number of molecules in any fixed volume is constant. The conservation of the number of molecules requires that

$$
d_{k k}+\Omega J_{k, k}=\Omega R \text {. }
$$

Even in the absence of injection, $R=0$, the divergence of the velocity field does not necessarily vanish. This behavior differs from that of hydrodynamics of incompressible fluids. Equation (8) is applicable to an inhomogeneous field, whereas (2) is applicable to a homogeneous field.

The body is subject to loads of several kinds (Fig. 5). Let $\gamma$ be the surface energy of the liquid per unit area. Associated with the change in the area $A$ of the surface of the body, the surface energy of the body changes at the rate $\gamma d A / d t$. The body is subject to external forces. Let $\mathbf{b}$ be the external force per unit volume in the body, and $\mathbf{t}$ be the external force per unit area on the surface of the body. Associated with the velocity of the markers, the potential energy of the external forces changes at the rate $-\int b_{i} v_{i} d V-\int t_{i} v_{i} d A$. Upon diffusing from the bulk to the surface, molecules are assumed to plate out on the surface, but do not leave the body. Associated with the molecules plated out, the surface of the body advances at velocity $\Omega J_{i} n_{i}$, the traction normal to the surface $t_{j} n_{j}$ does work, and the potential energy of the external forces changes at the rate $-\int t_{j} n_{j} \Omega J_{i} n_{i} d A$. Each small piece of the body may be in contact with a reservoir of the same species of molecules. Let $\mu$ be the chemical potential of molecules in the reservoir, and $R$ be the rate at which the reservoir injects molecules into the piece. The whole body can be connected with a field of reservoirs, so that both $\mu$ and $R$ are fields. Associated with the transfer of molecules from the reservoirs to the body, the Helmholtz free energy of the reservoirs 
changes at a rate $-\int \mu R d V$. The surface, the body, the external forces, and the reservoirs together constitute a composite thermodynamic system. Thermodynamics requires that the Helmholtz free energy of the composite system should never increase:

$$
\gamma \frac{d A}{d t}-\int b_{i} v_{i} d V-\int t_{i} v_{i} d A-\int t_{j} n_{j} \Omega J_{i} n_{i} d A-\int \mu R d V \leq 0
$$

Recall that we have set the Helmholtz free energy per molecule inside the liquid to be zero. The thermodynamic condition (9) holds for an inhomogeneous field, which should be compared to the thermodynamic condition for a homogeneous field (3).

Let $\kappa$ be the mean curvature of an element of the surface of the body. For example, the mean curvature of a spherical particle of radius $a$ is $\kappa=2 / a$, and the mean curvature of spherical cavity of radius $a$ is $\kappa=-2 / a$. Recall an identity in differential geometry: as the surface moves at velocity $\left(v_{i}+\Omega J_{i}\right) n_{i}$, the area of the surface changes at the rate $d A / d t=\int \kappa\left(v_{i}+\Omega J_{i}\right) n_{i} d A$. Pieces in the body communicate through the balance forces, $\sigma_{i j, j}+b_{i}=0 \quad, \quad$ giving $\int b_{i} v_{i} d V=\int \sigma_{i j} v_{i, j} d V-\int \sigma_{i j} n_{j} v_{i} d A$. Also note an identity $\int \mu J_{i, i} d V=\int \mu J_{i} n_{i} d A-\int \mu_{, i} J_{i} d V$

The above relations allow us to rewrite the thermodynamic condition (9) as

$$
\begin{aligned}
& \int\left(t_{i}-\sigma_{i j} n_{j}-\gamma \kappa n_{i}\right) v_{i} d A \\
& +\int\left(\mu+\Omega t_{j} n_{j}-\Omega \gamma \kappa\right) J_{i} n_{i} d A \\
& +\int\left(\sigma_{i j}+\frac{\mu}{\Omega} \delta_{i j}\right) v_{i, j} d V \\
& +\int-\mu_{i,} J_{i} d V \geq 0
\end{aligned}
$$

The inequality holds when the composite system is not in thermodynamic equilibrium, whereas the equality holds when the composite system is in thermodynamic equilibrium. The thermodynamic condition (10) holds for two independent and arbitrary fields: the velocity of 
markers, $\mathbf{v}$, and the self-diffusion flux of molecules, $\mathbf{J}$. Consequently, the integrand of each of the four integrals must be nonnegative.

We satisfy the four distinct conditions as follows. We assume local thermodynamic equilibrium at the surface of the body, so that the first two integrands vanish, namely,

$$
\begin{gathered}
\sigma_{i j} n_{j}=t_{i}-\gamma \kappa n_{i}, \\
\mu=-\Omega t_{j} n_{j}+\Omega \gamma \kappa .
\end{gathered}
$$

These equations represent four distinct boundary conditions. Whereas (11) expresses the balance of forces acting on the markers in the liquid near the surface, (12) expresses the condition of equilibrium as molecules in the interior of the liquid diffuse out and plate onto the surface, when no molecules leave the body or are added to the body from external reservoirs. In the absence of the surface energy, (11) recovers the familiar balance of forces on the surface, and (12) relates the chemical potential on the surface to the normal traction. In the absence of the traction due to external forces, (11) and (12) reduce to the expressions for the Laplace pressure and the Thompson effect [35]. The third integrand in (10) is nonnegative once we adopt the generalized model of viscous flow (Section 2). The fourth integrand in (10) is nonnegative once we adopt a model of self-diffusion, $J_{i}=-(D / \Omega k T) \mu_{, i}$, where $D$ is the self-diffusivity [36].

\section{Governing Equations and Characteristic Length and Time}

We now summarize the governing equations. Using the relation $d_{i j}=\left(v_{i, j}+v_{j, i}\right) / 2$, we write the generalized model of viscosity as

$$
\sigma_{i j}+\frac{\mu}{\Omega} \delta_{i j}=\eta\left(v_{i, j}+v_{j, i}\right)+\left(\beta-\frac{2 \eta}{3}\right) v_{k, k} \delta_{i j} .
$$

The balance of forces gives

$$
\sigma_{i j, j}+b_{i}=0
$$


The model of self-diffusion connects the self-diffusion flux to the gradient of the chemical potential:

$$
J_{i}=-\frac{D}{\Omega k T} \mu_{, i} .
$$

The molecular incompressibility, the conservation of the number of molecules, and the relation $d_{k k}=v_{k, k}$ together give that

$$
v_{k, k}+\Omega J_{k, k}=\Omega R .
$$

Equations (13)-(16) represent thirteen independent partial differential equations for the thirteen fields $v_{i}, \sigma_{i j}, J_{i}, \mu$.

Inserting (13) into (14), we obtain that

$$
\eta v_{i, k k}+\left(\frac{\eta}{3}+\beta\right) v_{k, k i}-\frac{\mu_{, i}}{\Omega}+b_{i}=0 .
$$

Inserting (15) into (16), we obtain that

$$
v_{k, k}-\frac{D}{k T} \mu_{, k k}=\Omega R .
$$

Equations (17) and (18) represent four distinct partial differential equations for the four fields, $v_{i}, \mu$. These four differential equations are solved along with the four independent boundary conditions (11) and (12).

We will be usually interested in problems with vanishing body force $\mathbf{b}$ and vanishing rate of injection $R$. In the latter case, the body is disconnected from reservoirs of molecules. The value of $\mu$ at a particular piece in the body can be interpreted by the following operation. When we connect the piece in the body to a reservoir, $\mu$ is the chemical potential of the molecules in the reservoir needed to equilibrate with the piece. Thus the reservoir serves as an instrument that measures chemical potential, analogous to a thermometer that measures temperature.

A comparison of (17) and (18) gives a length [34] 


$$
\Lambda=\sqrt{\frac{\eta D \Omega}{k T}} .
$$

The length characterizes the relative rate of viscous flow and self-diffusion. For any given boundary-value problem, the geometry of the body specifies additional lengths. Let $a$ be a length representative of the geometry of the body. In the limit $a>>\Lambda$, self-diffusion is negligible, (18) reduces to $v_{k, k}=0$, and (17) recovers the Stokes equations for viscous flow [31]. In the limit $a<\Lambda$, viscous flow is negligible, (18) reduces to the equation for self-diffusion [36].

The characteristic length $\Lambda$ is material-specific (Fig. 6a). Note that the quantity $\eta D / k T$ appears both in the equation defining the characteristic length (19) and in the Stokes-Einstein relation (1). For a liquid obeying the Stokes-Einstein relation, such as silica, viscous flow and self-diffusion result from the single rate-limiting process-molecules change neighbors, so that the length $\Lambda$ is of molecular size and is independent of temperature. For a liquid violating the Stokes-Einstein relation, however, viscous flow and self-diffusion result from different ratelimiting processes, so that the length $\Lambda$ can be much larger than the molecular size and increases as temperature drops.

We also identify a characteristic time:

$$
\Gamma=\eta \Lambda / \gamma
$$

This time is also material-specific and temperature-dependent (Fig. 6b). The surface energy $\gamma$ typically depends on temperature weakly. For a liquid obeying the Stokes-Einstein relation, the length $\Lambda$ is independent of temperature, and the time $\Gamma$ follows the Arrhenius behavior of viscosity. By contrast, for a liquid violating the Stokes-Einstein relation, the length $\Lambda$ may be much larger than the size of molecules, and the viscosity exhibits non-Arrhenius behavior. Consequently, the plot of the time $\log \Gamma$ as a function of $1 / T$ curves up significantly.

\section{Shrinking of a Cavity in a Supercooled Liquid}


We apply our theory to a cavity in an infinite body of supercooled liquid (Fig. 7). Under the influence of the surface energy, the cavity shrinks by concurrent viscous flow and selfdiffusion. The cavity is spherical, so that the non-vanishing fields are the radial component of stress $\sigma_{r}$, circumferential component of stress $\sigma_{\theta}$, chemical potential $\mu$, radial component of the marker velocity $v$, and the radial component of the self-diffusion flux $J$. For the cavity at a given radius $a$, all these fields are functions of radial coordinate $r$. In this illustration, we neglect the effect of the bulk viscosity and set $\beta=0$, so that $\left(\sigma_{r}+2 \sigma_{\theta}\right) / 3+\mu / \Omega=0$. We use this equation to express $\sigma_{\theta}$ in terms of $\sigma_{r}$ and $\mu$. Note that $d_{r}=d v / d r$ and $d_{\theta}=v / r$, so that the kinetic model of viscous flow (6) reduces to

$$
\sigma_{r}=\frac{4}{3} \eta\left(\frac{d v}{d r}-\frac{v}{r}\right)-\frac{\mu}{\Omega} .
$$

The balance of forces requires that

$$
\frac{d \sigma_{r}}{d r}+\frac{2}{r}\left(\sigma_{r}-\sigma_{\theta}\right)=0
$$

The self-diffusion flux is linear in the gradient of the chemical potential:

$$
J=-\frac{D}{\Omega k T} \frac{d \mu}{d r}
$$

The molecular incompressibility takes a simple form under the spherical symmetry. Here we assume that the liquid is disconnected from any reservoir of molecules. Denote the radius of cavity at time $t$ by $a(t)$. At a given time, the net flow of molecules crossing the surface of the cavity is $4 \pi a^{2}(d a / d t) / \Omega$, and the net flow of molecules crossing the spherical surface of radius $r$ is $4 \pi r^{2}(J(r)+v(r) / \Omega)$. The molecular incompressibility requires that the two flows should be equal, giving that

$$
v(r)+\Omega J(r)=\left(\frac{a}{r}\right)^{2} \frac{d a}{d t}
$$


We use this equation to express $v(r)$ in terms of $J(r)$.

Equations (21)-(23) are three first-order ordinary differential equations for the three functions $\sigma_{r}(r), \mu(r), J(r)$, in the interval $r \in(a, \infty)$, subject to the following boundary conditions. The surface of the cavity is concaved and has a negative mean curvature, $\kappa=-2 / a$. No external traction is applied on the surface of the cavity, so that the boundary conditions (11) and (12) become $\sigma_{r}(a)=2 \gamma / a$ and $\mu(a)=-2 \Omega \gamma / a$. Far from the cavity, the radial stress and the chemical potential vanish: $\sigma_{r}(\infty)=0$ and $\mu(\infty)=0$.

For a given radius $a$, we solve the three coupled ordinary-differential equations numerically using the shooting method. Specifically, we assume some values of $J(a)$ and $d a / d t$, use the two boundary conditions $\sigma_{r}(a)=2 \gamma / a$ and $\mu(a)=-2 \Omega \gamma / a$, and numerically integrate the three ordinary differential equations (21)-(23). We adjust the values of $J(a)$ and $d a / d t$ to satisfy the boundary conditions $\sigma_{r}(\infty)=0$ and $\mu(\infty)=0$. We plot the shrinking rate of the cavity as a function of the radius of the cavity (Fig. 7).

In the large-cavity limit, $a>>$, the cavity shrinks by viscous flow, and molecular incompressibility (24) reduces to $v(r)=(a / r)^{2} d a / d t$. Newton's law of viscosity applies, $\sigma_{r}-\sigma_{\theta}=2 \eta\left(d_{r}-d_{\theta}\right)=-6 \eta a^{2} r^{-3} d a / d t$. Inserting this expression into the equation of the balance of forces $d \sigma_{r} / d r+2\left(\sigma_{r}-\sigma_{\theta}\right) / r=0$, integrating over the interval $r \in(a, \infty)$, and using the boundary conditions $\sigma_{r}(a)=2 \gamma / a$ and $\sigma_{r}(\infty)=0$, we obtain that

$$
\frac{d a}{d t}=-\frac{\gamma}{2 \eta}
$$

This expression agrees with the numerical solution of concurrent viscous flow and self-diffusion in the limit of large cavities (Fig. 7). 
In the small-cavity limit, $a<<\Lambda$, the cavity shrinks by self-diffusion, and molecular incompressibility (24) reduces to $\Omega J(r)=(a / r)^{2} d a / d t$. Inserting this expression into the model of self-diffusion $J=-(D / \Omega k T) d \mu / d r$, integrating over the interval $r \in(a, \infty)$, and using the boundary conditions $\mu(a)=-2 \Omega \gamma / a$ and $\mu(\infty)=0$, we obtain that

$$
\frac{d a}{d t}=-\frac{2 D \gamma \Omega}{k T a^{2}} .
$$

This expression agrees with the numerical solution of concurrent viscous flow and self-diffusion in the limit of small cavities (Fig. 7).

The transition from shrinking mediated by self-diffusion to that mediated by viscous flow takes place when the radius of the cavity is comparable to the length $\Lambda$, and when the time of observation is on the scale of $\Gamma$ (Fig. 7). For a liquid that obeys the Stokes-Einstein relation, such as silica, the length $\Lambda$ is of molecular dimension (Fig. 6a), so that a cavity of any size larger than individual molecules shrinks by viscous flow. For a liquid that violates the Stokes-Einstein relation, the length $\Lambda$ can be much larger that molecular dimension as the temperature drops (Fig. 6a), so that a small enough cavity shrinks by self-diffusion.

\section{Discussion}

At a given temperature, a supercooled liquid forms dynamic structure of some length scale $\xi$ [27]. The dynamic structure has a lifetime $\tau$, after which the correlation within the structure disappears [37-39]. Any continuum theory should be interpreted with care when the geometric length and the time scale is close to, or smaller than, $\xi$ and $\tau$. The exact connection between this molecular picture and our continuum theory remains open.

We can compare our theory with the classical hydrodynamics. Our theory regards viscous flow and self-diffusion as concurrent, but distinct, kinetic processes. We describe the kinematics of a liquid by two independent fields: the velocity of markers, $\mathbf{v}$, and the net flux of 
molecules, $\mathbf{N}$. Both fields can be determined in macroscopic experiments. We define the selfdiffusion flux by $\mathbf{J}=\mathbf{N}-\mathbf{v} / \Omega$. We prescribe kinetic models for viscous flow and self-diffusion separately. By contrast, in the classical hydrodynamics, the Navier-Stokes equations govern the velocity of the center of mass $\mathbf{v}_{m}$, which relates to the net flux of molecules by $\mathbf{v}_{m}=\Omega \mathbf{N}$, leaving no room for self-diffusion to contribute to the governing equations [31]. The Navier-Stokes equations use Newton's law of viscous flow, and the Stokes-Einstein relation links the viscosity and self-diffusivity. As we have noted, experiments in recent decades have shown that the Stokes-Einstein relation is invalid for many supercooled liquids (Section 1).

For a body in three dimensions, our theory requires four independent boundary conditions. We have so far given boundary conditions that describe a surface under the influence of traction and surface tension, (11) and (12). We can also apply boundary conditions of other types. For example, for a rigid, impermeable wall, we may prescribe the boundary conditions $\mathbf{v}=0$ and $J_{i} n_{i}=0$. The former is the same as the no-slip, no-penetration boundary conditions in classical hydrodynamics. The latter represents the condition that molecules in the interior of the liquid cannot plate out onto the surface, or vice versa. This boundary condition has been used to analyze concurrent electromigration and creep in solders [40].

The kinetic model (6) is reminiscent of Biot's model of poroelasticity [41]. In recent years, Biot's model has been adapted to analyze gels, where molecules are assumed to be incompressible [42-44]. The kinetic model is analogous to the model of poroelasticity once we replace the rate of deformation with strain, and replace the two viscosities with the two elastic moduli. In particular, when molecules are assumed to be incompressible, the bulk modulus in poroelasticity has nothing to do with elastic compressibility, but represents the ability of the material to absorb additional solvent under hydrostatic tensile stress [44]. In analogy with Biot's model of poroviscosity, we may call the kinetic model (6) a model of poroviscosity. 
Our continuum theory of supercooled liquids is closely related to concurrent creep and self-diffusion in solids [34, 41]. We have neglected elasticity and adopted the simplest kinetic models. However, supercooled liquids may exhibit viscoelasticity. Our theory can be extended to describe concurrent self-diffusion and viscoelastic flow, similar to previous theories for amorphous systems $[45,46]$. One could for instance adopt a Maxwell-type viscoelasticity model with multiple relaxation times. We have also adopted a linear relation between the self-diffusion flux and the gradient of chemical potential, with a constant self-diffusivity. However, several authors reported the existence of crossover length (which scales with $\xi$ ) and time below which the self-diffusivity varying with the length of the observation [27, 29, 47]. As previously mentioned, our theory is not intended to analyze phenomena at a length below the size of the dynamic heterogeneity and a time shorter than the lifetime of the dynamic heterogeneity.

\section{Concluding Remarks}

As the temperature drops, a supercooled liquid may be partially jammed: viscous flow slows down greatly, but self-diffusion does not slow down as much. We regard viscous flow and self-diffusion as distinct processes, and formulate a continuum theory to evolve a body of such a liquid under the influence of external forces, surface tension, and reservoirs of molecules. Because small pieces of the liquid exchange molecules and do not retain identities, we describe kinematics not by tracking the small pieces of the liquid, but by tracking the velocity of markers and the net flux of molecules. We describe viscous flow and self-diffusion using two independent kinetic models. The relative rate of the two kinetic processes defines a characteristic length that demarcates two types of behavior. Large objects evolve by viscous flow, and small objects evolve by self-diffusion. It is hoped that an experimental system will soon be identified to demonstrate this transition. Concurrent diffusion and viscous flow should also be important in other partially jammed systems, such as gels consisting of macromolecules and small-molecule solvents, and glasses consisting of dissimilar atoms. 


\section{ACKNOWLEDGMENT}

This work at Harvard is supported by MRSEC (DMR-0820484). Li is supported by China Scholarship Council as a visiting scholar for two years at Harvard University. Brassart is supported by the National Fund for Research of Belgium (FNRS).

\section{REFERENCES}

[1] Dienes, G. J., and Klemm, H. F., 1946, "Theory and application of the parallel plate plastometer,” J. Appl. Phys., 17, pp. 458-471. http://dx.doi.org/10.1063/1.1707739

[2] Cotts, R. M., Hoch, M. J. R., Sun, T., and Markert, J. T., 1989, "Pulsed field gradient stimulated echo methods for improved NMR diffusion measurements in heterogeneous systems,” J. Magn. Reson., 83, pp. 252-266. DOI: 10.1016/oo22-2364(89)90189-3

[3] Smith, R. S., Dohnálek, Z., Kimmel, G. A., Stevenson, K. P., and Kay, B. D., 200o, “The selfdiffusivity of amorphous solid water near 150K," Chem. Phys., 258, pp. 291-305. DOI: 10.1016/So301-0104(00)00130-0

[4] Mills, P. J., Green, P. F., PalmstrØm, C. J., Mayer, J. W., and Kramer, E. J., 1984, “Analysis of diffusion in polymers by forward recoil spectrometry," Appl. Phys. Lett., 45, pp. 957-959. http://dx.doi.org/10.1063/1.95456

[5] Einstein, A., 1905, Uber die von der molekularkinetischen theorie der warme geforderte bewegung von in ruhenden flussigkeiten suspendierten teilchen. English translation: On the movement of small particles suspended in a stationary liquid demanded by the molecular-kinetic theory of heat, in Investigations on the Theory of the Brownian Movement, Dover, New York, 1956.

[6] Edward, J. T., 1970, "Molecular volumes and the Stokes-Einstein equation,” J. Chem. Educ., 47(4), pp.261-270. DOI: 10.1021/edo47p261 
[7] Borucka, A. Z., Bockris, J. O’M., and Kitchener, J. A., 1957, “Self-Diffusion in Molten Sodium Chloride: A Test of the Applicability of the Nernst-Einstein Equation,” Proc. R. Soc. A, 241(1227), pp. 554-567. http://www.jstor.org/stable/2413792

[8] Ediger, M. D., Angell, C. A., and Nagel, S. R., 1996, “Supercooled liquids and glasses,” J. Phys. Chem., 10o, pp. 13200-13212. DOI: 10.1021/jp953538d

[9] Rosenfeld, D., and Woodley, W. L., 200o, “Deep convective clouds with sustained supercooled liquid water down to $-37.5{ }^{\circ} \mathrm{C}$," Nature, 405, pp. 440-442. DOI: $10.1038 / 35013030$

[10] Shi, Z., Debenedetti, P. G., and Stillinger, F. H., 2013, "Relaxation processes in liquids: variations on a theme by Stokes and Einstein,” J. Chem. Phys., 138, 12 A526. http://dx.doi.org/10.1063/1.4775741

[11] Angell, C. A., Ngai, K. L., McKenna, G. B., McMillan, P. F., and Martin, S. W., 200o, "Relaxation in glassforming liquids and amorphous solids," Appl. Phys. Rev., 88, pp. 3113 3157. http://dx.doi.org/10.1063/1.1286035

[12] Debenedetti, P. G., and Stillinger, F. H., 2001, "Supercooled liquids and the glass transition," Nature, 410, pp. 259-267. DOI: 10.1038/35065704

[13] Mapes, M. K., Swallen, S. F., and Ediger, M. D., 2006, "Self-diffusion of supercooled oterphenyl near the glass transition temperature,” J. Phys. Chem. B, 11o, pp. 507-511. DOI: 10.1021/jp0555955

[14] Swallen, S. F., Mapes, M. K., Kim, Y. S., McMahon, R. J., Ediger, M. D., and Satija, S., 20o6, "Neutron reflectivity measurements of the translational motion of tris(naphthylbenzene) at the glass transition temperature,” J. Chem. Phys., 124, pp. 184501. http://dx.doi.org/10.1063/1.2191492

[15] Swallen, S. F., Traynor, K., McMahon, R. J., Ediger, M. D., and Mates, T. E., 2009, "Selfdiffusion of supercooled tris-naphthylbenzene,” J. Phys. Chem. B, 113, pp. 4600-4608. DOI: 10.1021/jp808912e 
[16] Fujara, F., Geil, B., Sillescu, H., and Fleischer, G., 1992, “Translational and rotational diffusion in supercooled orthoterphenyl close to the glass transition,” Z. Phys. B Condensed Matter, 88, pp. 195-204. DOI: 10.1007/BF01323572

[17] Cavagna, A., 2009, “Supercooled liquids for pedestrians," Phys. Rep., 476, pp. 51-124. DOI: 10.1016/j.physrep.2009.03.003

[18] Ediger, M. D., and Harrowell, P., 2012, "Perspective: supercooled liquids and glasses," J. Chem. Phys., 137, 080901. http://dx.doi.org/10.1063/1.4747326

[19] Ediger, M. D., 200o, “Spatially heterogeneous dynamics in supercooled liquids,” Annu. Rev. Phys. Chem., 51, pp. 99-128. DOI: 10.1146/annurev.physchem.51.1.99

[20] Berthier, L., 2011, “Dynamic heterogeneity in amorphous materials,” Physics, 4, pp. 42. DOI: $10.1103 /$ Physics.4.42

[21] Karmakar, S., Dasgupta, C., and Sastry, S., 2014, "Growing length scales and their relation to timescales in glass-forming liquids,” Annu. Rev. Condens. Matter Phys., 5, pp. 255-284. DOI: 10.1146/annurev-conmatphys-031113-133848

[22] Sillescu, H., Böhmer, R., Diezemann, G., and Hinze, G., 2002, "Heterogeneity at the glass transition: what do we know?” J. Non-Cryst. Solids, 307-310, pp. 16-23. DOI: 10.1016/Soo22-3093(02)01435-7

[23] Reinsberg, S. A., Qiu, X. H., Wilhelm, M., Spiess, H. W., and Ediger, M. D., 2001, "Length scale of heterogeneous dynamic heterogeneity in supercooled glycerol near $\mathrm{T}_{\mathrm{g}}$, J. Chem. Phys., 114, pp. 7299-7302. http://dx.doi.org/10.1063/1.136916o

[24] Qiu, X. H., and Ediger, M. D., 2003, "Length scale of dynamic heterogeneity in supercooled D-Sorbitol: comparison to model predictions,” J. Phys. Chem. B, 1o7, pp. 459-464. DOI: 10.1021/jpo21888b

[25] Berthier, L., Biroli, G., Bouchaud, J.-P., Cipelletti, L., Masri, D. El, L’Hôte, D., Ladieu, F., and Pierno, M., 2005, “Direct experimental evidence of a growing length scale 
accompanying the glass transition," Science, 310, pp. 1797-1800. DOI:

10.1126/science.1120714

[26] Furukawa, A., and Tanaka, H., 2011, "Direct evidence of heterogeneous mechanical relaxation in supercooled liquids,” Phys. Rev. E, 84, 061503. http://dx.doi.org/10.1103/PhysRevE.84.061503

[27] Furukawa, A., 2013, "Simple picture of supercooled liquid dynamics: dynamic scaling and phenomenology based on clusters," Phys. Rev. E, 87, 062321. http://dx.doi.org/10.1103/PhysRevE.87.062321

[28] Yamamoto, R., and Onuki, A., 1998, "Heterogeneous diffusion in highly supercooled liquids,” Phys. Rev. Lett., 81, pp. 4915-4918. http://dx.doi.org/10.1103/PhysRevLett.81.4915

[29] Berthier, L., Chandler, D., and Garrahan, J. P., 2005, "Length scale for the onset of Fickian diffusion in supercooled liquids,” Europhys. Lett., 69, pp. 320-326. DOI: 10.1209/epl/i2004-10401-5

[30] Kumar, S. K., Szamel, G., and Douglas, J. F., 2006, "Nature of the breakdown in the StokesEinstein relationship in a hard sphere fluid,” J. Chem. Phys., 124, 214501. http://dx.doi.org/10.1063/1.2192769

[31] Batchelor, G. K., 1967, An introduction to fluid dynamics, Cambridge University Press.

[32] Darken, L. S., 1948, "Diffusion, mobility and their interrelation through free energy in binary metallic systems," Transactions of the American Institute of Mining and Metallurgical Engineers, 175, pp. 184-201.

[33] Stephenson, G. B., 1988, “Deformation during interdiffusion,” Acta Metall., 36, pp. 26632683. DOI: 10.1016/0001-616o(88)90114-9

[34] Suo, Z., 2004, “A continuum theory that couples creep and self-diffusion,” J. Appl. Mech., 71, pp. 646-651. DOI: 10.1115/1.1781176 
[35] Mullins, W. W., 1959, "Flattening of a nearly plane solid surface due to capillarity,” J. Appl. Phys., 3o, pp. 77-83. DOI: 10.1063/1.1734979

[36] Herring, C., 1950, “Diffusional viscosity of a polycrystalline solid,” J. Appl. Phys., 21, pp. 437-445. DOI: 10.1063/1.1699681

[37] Tracht, U., Wilhelm, M., Heuer, A., Feng, H., Schmidt-Rohr, K., and Spiess, H. W., 1998, “Length Scale of Dynamic Heterogeneities at the Glass Transition Determined by Multidimensional Nuclear Magnetic Resonance,” Phys. Rev. Lett., 81, pp. 2727-2730. http://dx.doi.org/10.1103/PhysRevLett.81.2727

[38] Graessley, W. W., 2009, “On dynamic heterogeneity in supercooled liquids,” J. Chem. Phys., 130, 164502. http://dx.doi.org/10.1063/1.3119641

[39] Russel, E. V., and Israeloff, N.E., 2000, “Direct observation of molecular cooperativity near the glass transition,” Nature, 408, pp.695-698 DOI: 10.1038/35047037

[40] Pharr, M., Zhao K., Suo, Z., Ouyang, F., and Liu, P., 2011, “Concurrent electromigration and creep in lead-free solder,” J. Appl. Phys., 11o, o83716. http://dx.doi.org/10.1063/1.3656002

[41] Biot, M. A., 1941, “General theory of three-dimensional consolidation,” J. Appl. Phys., 12, pp. 155-164. http://dx.doi.org/10.1063/1.1712886

[42] Hui, C. Y., Lin, Y. Y., Chuang, F. C., Shull, K. R., and Lin, W. C., 2006, “A contact mechanics method for characterizing the elastic properties and permeability of gels,” J. Polym. Sci. Part B Polym. Phys., 44, pp.359-370. DOI: 10.1002/polb.20613

[43] Galli, M., Comley, S. C., Shean, T. A. V., and Oyen, M. L., 2009, "Viscoelastic and poroelastic mechanical characterization of hydrated gels,” J. Mater. Res., 24, pp. 973-979. http://dx.doi.org/10.1557/jmr.2009.0129

[44] Yoon, J., Cai, S., Suo, Z., and Hayward, R. C., 2010, "Poroelastic swelling kinetics of thin hydrogel layers: comparison of theory and experiment," Soft Matter, 6, pp. 6004-6012. DOI: $10.1039 /$ CoSMoo434K 
[45] Hu Y., and Suo, Z., 2012, "Viscoelasticity and poroelasticity in elastomeric gels," Acta Mech. Solida Sin., 25, pp. 441-458. DOI: 10.1016/So894-9166(12)60039-1

[46] Brassart, L., and Suo, Z., 2013, "Reactive flow in solids," J. Mech. Phys. Solids, 61, pp. 61-77. DOI: 10.1016/j.jmps.2012.09.007

[47] Szamel, G., and Flenner, E., 2006, "Time scale for the onset of Fickian diffusion in supercooled liquids," Phys. Rev. E, 73, 011504. DOI: 10.1103/PhysRevE.73.011504

[48] Chang, I., and Sillescu, H., 1997, "Heterogeneity at the Glass Transition Translational and Rotational Self-Diffusion,” J. Phys. Chem. B, 101, pp. 8794-8801. DOI: 10.1021/jp9640989

[49] Swallen, S. F., and Ediger, M. D., 2011, "Self-diffusion of the amorphous pharmaceutical indomethacin near T,," Soft Matter, 7, pp. 10339-10344. DOI: 10.1039/C1SMo6283B

[50] Royal Society of Chemistry, ChemSpider Online Database, http://www.chemspider.com/

[51] Kingery, W. D., 1959, "Surface Tension of Some Liquid Oxides and Their Temperature Coefficients," J. Am. Ceram. Soc., 42(1), pp. 6-10. DOI: 10.1111/j.1151-2916.1959.tbo9134.x 

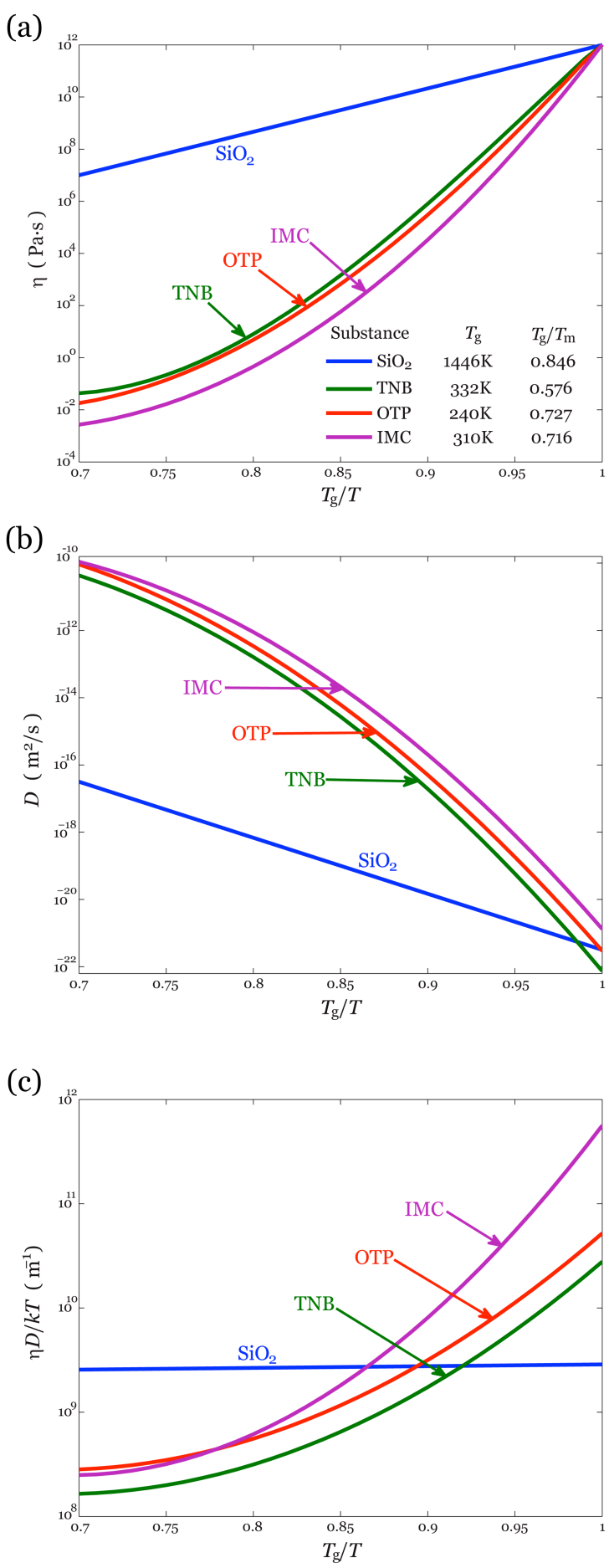

Fig. 1 Arrhenius plots of (a) viscosity $\eta$, (b) self-diffusivity $D$, and (c) the quantity $\eta D / k T$. Here $T$ is the temperature, $T_{m}$ is the melting point, and $T_{g}$ is the glass transition temperature defined as the temperature at which the viscosity of a given substance reaches $\eta=10^{12} \mathrm{~Pa} \cdot \mathrm{s}$. Included are data for several substances [48-50]: silica, 1,3-bis-(1-naphthyl)-5-(2naphthyl)benzene (TNB), o-terphenyl (OTP) and 1-(p-chlorobenzoyl)-5-methoxy-2-methylindole-3-acetic acid (IMC). 


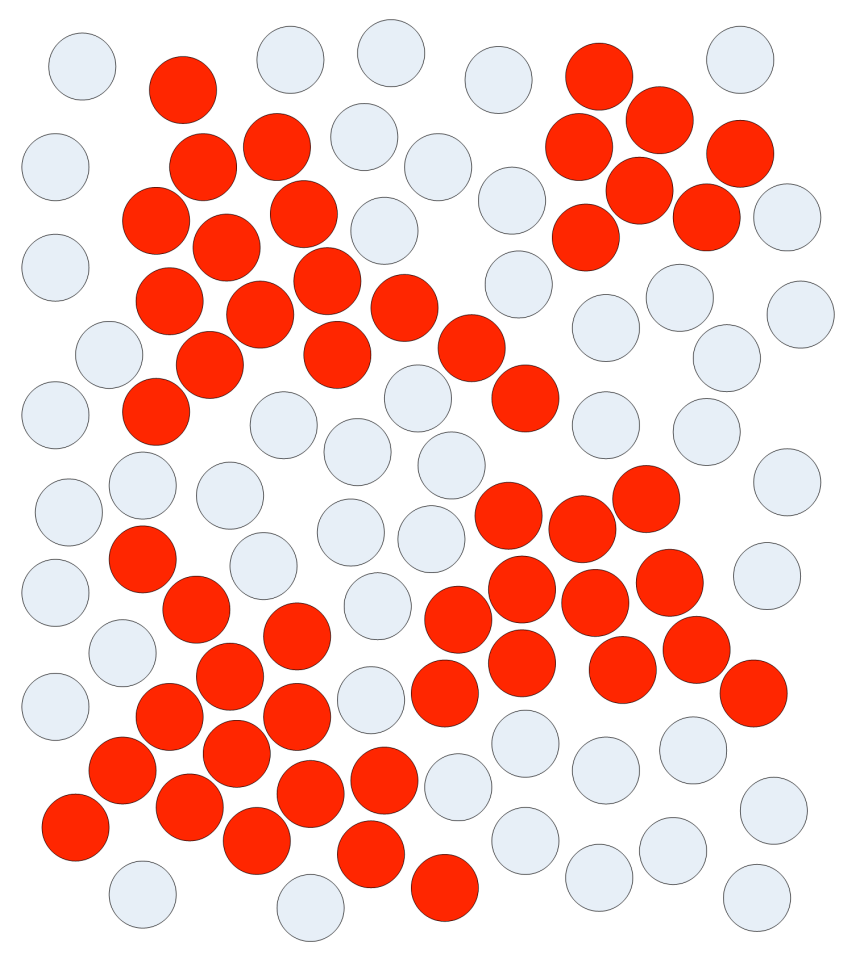

Fig. 2 A supercooled liquid may form a dynamic structure, consisting of regions in which molecules rearrange at very different rates. Here we represent slow regions in red, and fast regions in grey. The size of these regions is large compared to the size of individual molecules. Viscous flow proceeds by disrupting the dynamic structure, but self-diffusion proceeds as individual molecules migrate through the liquid. As the temperature drops, the dynamic structure increasingly jams viscous flow, but does not retard self-diffusion as much. 


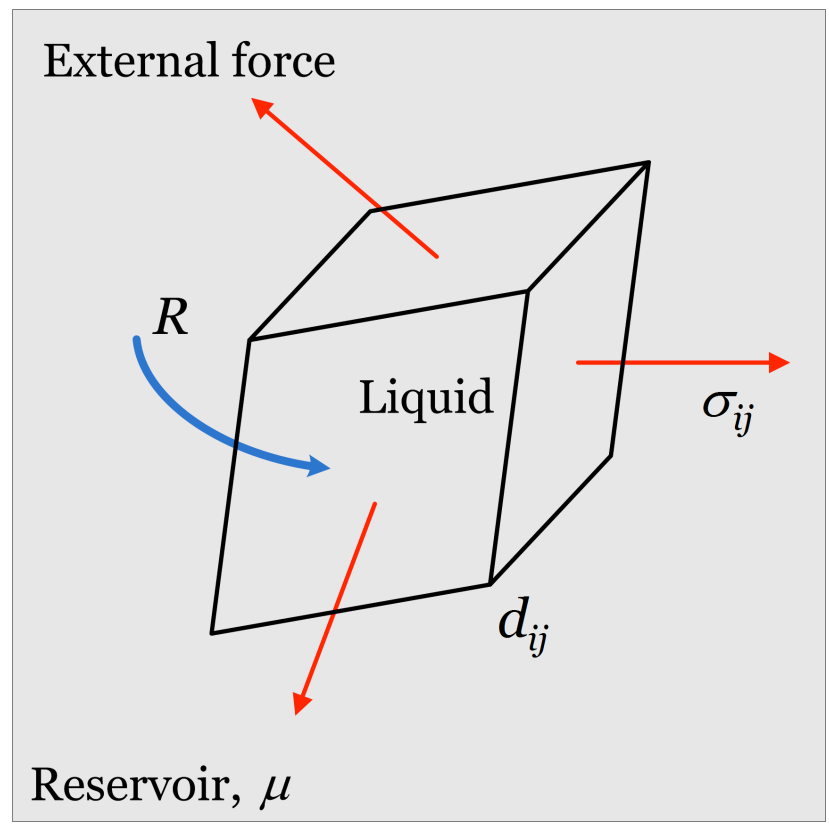

Fig. 3 A composite thermodynamic system consists of a piece of liquid, a set of external forces, and a reservoir of molecules. The piece evolves through a sequence of homogeneous states, represented by a parallelepiped that changes its shape and volume at a rate of deformation $d_{i j}$, and absorbs molecules from the reservoir at rate $R$ per unit volume. The external forces apply to the piece of liquid a state of stress $\sigma_{i j}$. The reservoir and the piece of liquid exchange the species of molecules that constitute the liquid, and the chemical potential of the species of molecules in the reservoir is $\mu$. 


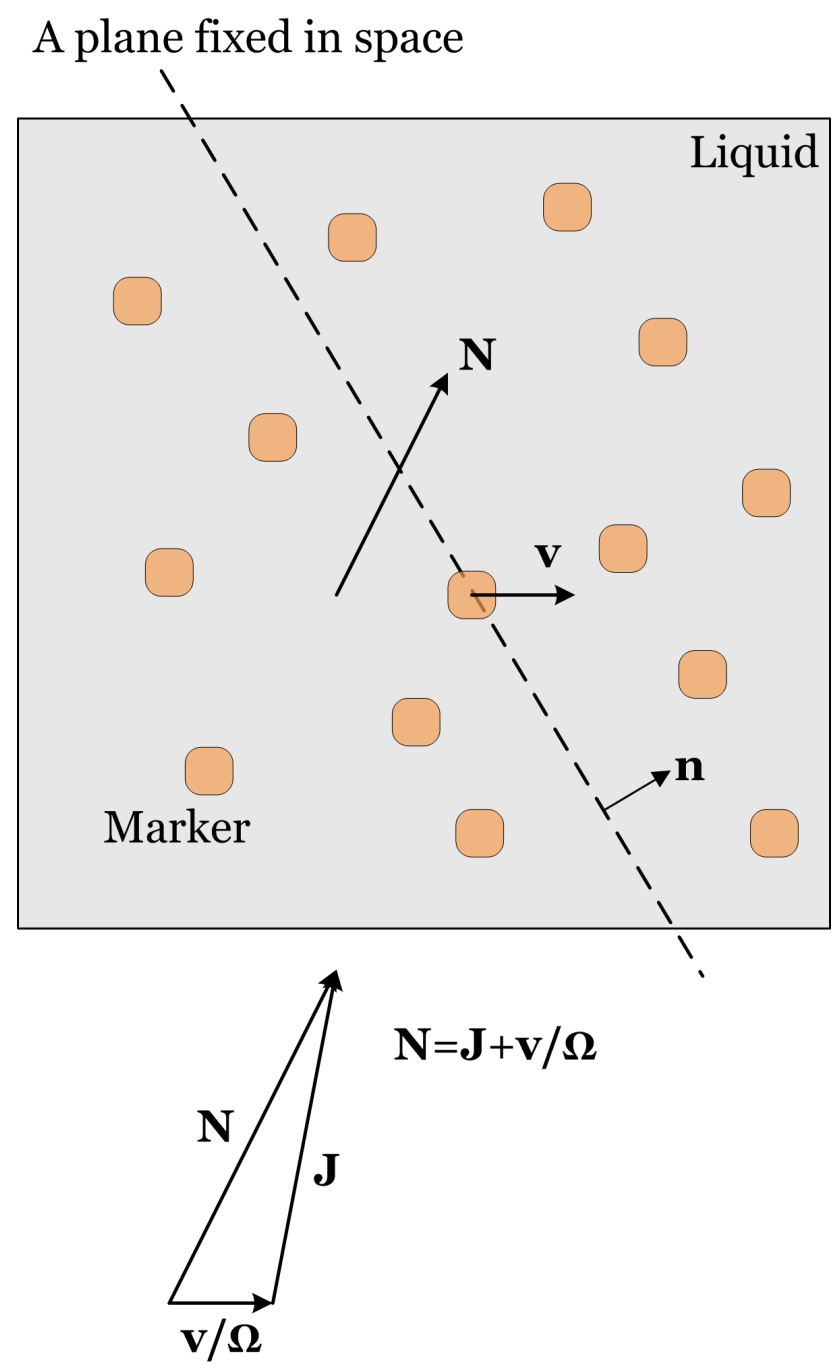

Fig. 4 The definition of self-diffusion flux using the net flux of molecules and the velocity of markers. Imagine a plane fixed in space, with $\mathbf{n}$ being the unit vector normal to the plane. The net flux of molecules $\mathbf{N}$ is a vector, such that $\mathbf{N} \cdot \mathbf{n}$ is the number of molecules crossing the plane per unit area and per unit time. Dispersed in the liquid are markers moving at the velocity $\mathbf{v}$. The net flux of molecules and the velocity of markers can be independently measured. Define the self-diffusion flux by $\mathbf{J}=\mathbf{N}-\mathbf{v} / \Omega$, where $\Omega$ is the volume per molecule. 


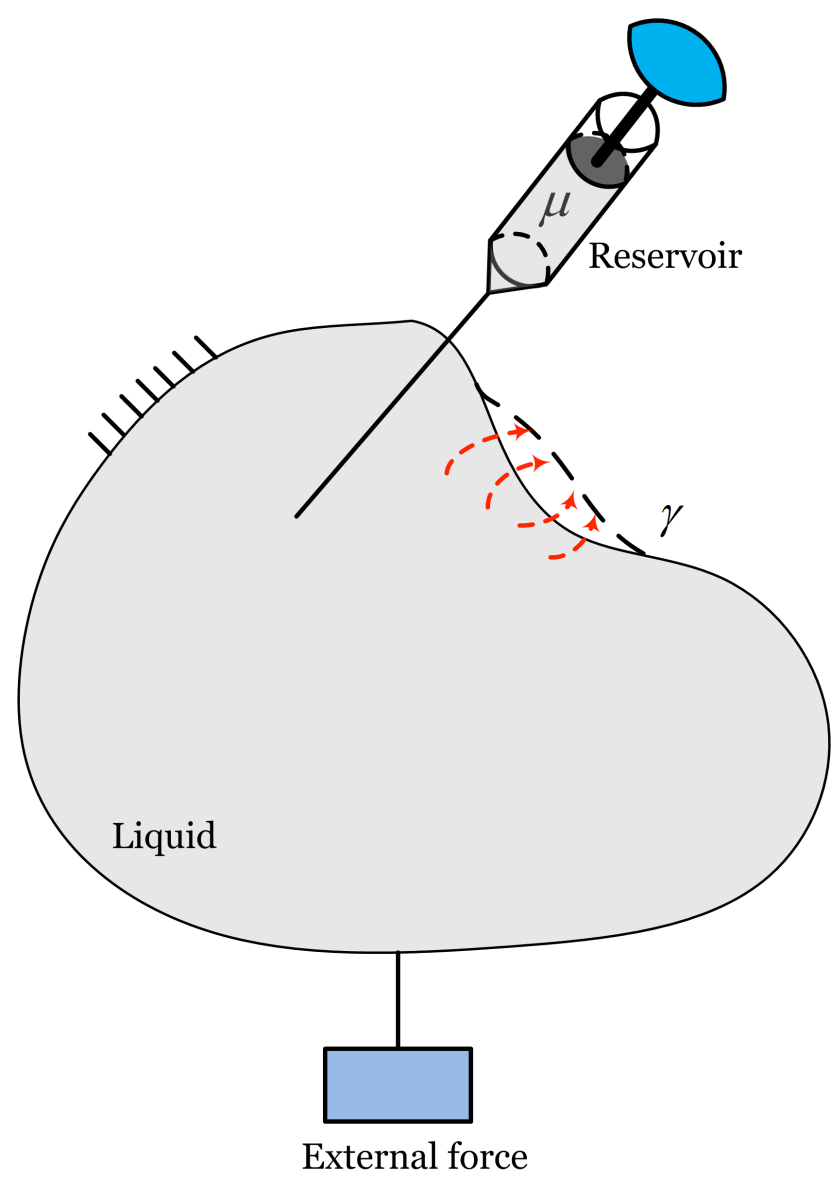

Fig. 5 A composite thermodynamic system consists of a body of a pure liquid, the surface of the body, a set of external forces, and a set of reservoirs. The reservoirs can inject molecules of the same species as those constitute the liquid. Molecules in the interior of the body can diffuse out and plate onto the surface, but cannot leave the liquid. As the surface of the body moves, the area of the surface changes, and the total surface energy changes. 
Journal of Applied Mechanics 81, 111007 (2014)

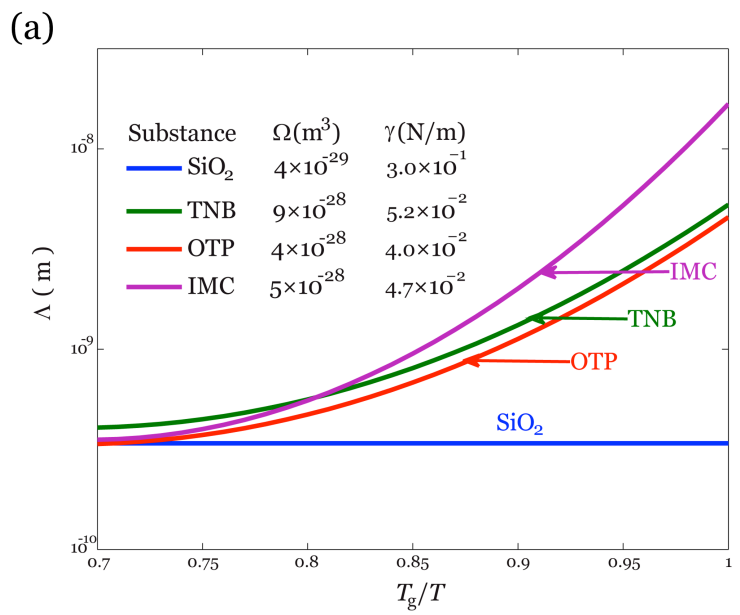

(b)

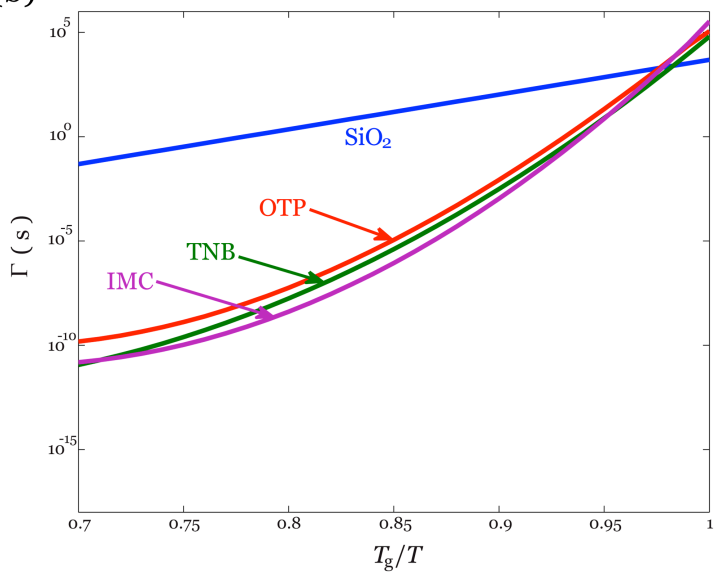

Fig. 6 Arrhenius plots of (a) the characteristic length $\Lambda$, and (b) the characteristic time $\Gamma$. Here $T$ is the temperature, and $T_{g}$ is the glass transition temperature. Data are from [48-51]. 


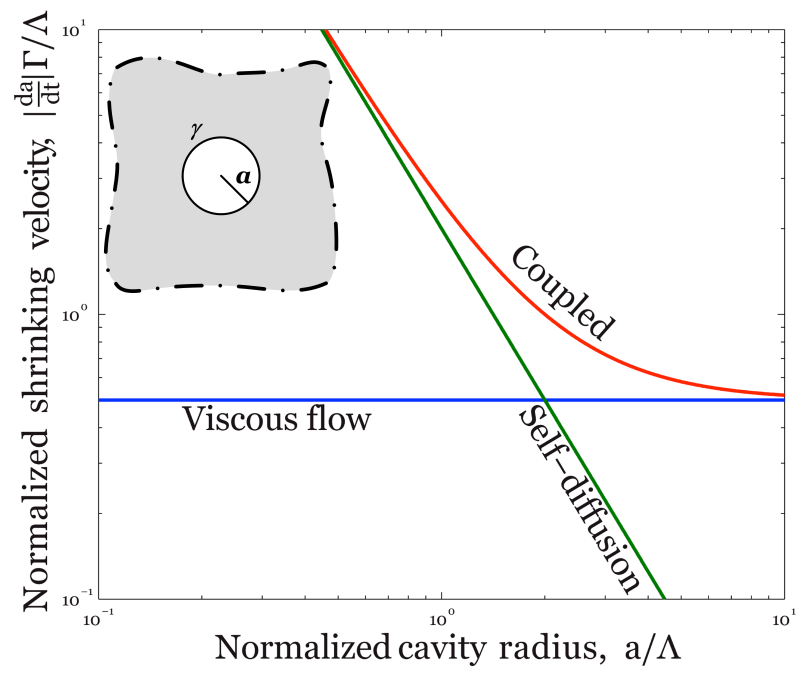

Fig. 7 In a supercooled liquid, a spherical cavity shrinks under the influence of surface energy. The rate of shrinking is a function of the radius of the cavity. A small cavity shrinks by selfdiffusion, a large cavity shrinks by viscous flow, and a cavity of an intermediate size shrinks by coupled self-diffusion and viscous flow. 